\title{
The association between malnutrition and the incidence of malaria among young HIV-infected and -uninfected Ugandan children: a prospective study
}

Emmanuel Arinaitwe ${ }^{1 *}$, Anne Gasasira ${ }^{1}$, Wendy Verret $^{2}$, Jaco Homsy ${ }^{3}$, Humphrey Wanzira', Abel Kakuru', Taylor G Sandison ${ }^{4}$, Sera Young ${ }^{5}$, Jordan W Tappero ${ }^{6}$, Moses R Kamya ${ }^{7}$ and Grant Dorsey ${ }^{8}$

\begin{abstract}
Background: In sub-Saharan Africa, malnutrition and malaria remain major causes of morbidity and mortality in young children. There are conflicting data as to whether malnutrition is associated with an increased or decreased risk of malaria. In addition, data are limited on the potential interaction between HIV infection and the association between malnutrition and the risk of malaria.

Methods: A cohort of 100 HIV-unexposed, 203 HIV-exposed (HIV negative children born to HIV-infected mothers) and 48 HIV-infected children aged 6 weeks to 1 year were recruited from an area of high malaria transmission intensity in rural Uganda and followed until the age of 2.5 years. All children were provided with insecticidetreated bed nets at enrolment and daily trimethoprim-sulphamethoxazole prophylaxis (TS) was prescribed for HIVexposed breastfeeding and HIV-infected children. Monthly routine assessments, including measurement of height and weight, were conducted at the study clinic. Nutritional outcomes including stunting (low height-for-age) and underweight (low weight-for-age), classified as mild (mean z-scores between -1 and -2 during follow-up) and moderate-severe (mean z-scores $<-2$ during follow-up) were considered. Malaria was diagnosed when a child presented with fever and a positive blood smear. The incidence of malaria was compared using negative binomial regression controlling for potential confounders with measures of association expressed as an incidence rate ratio (IRR).
\end{abstract}

Results: The overall incidence of malaria was 3.64 cases per person year. Mild stunting (IRR $=1.24,95 \% \mathrm{Cl} 1.06-$ $1.46, \mathrm{p}=0.008)$ and moderate-severe stunting (IRR $=1.24,95 \% \mathrm{Cl} 1.03-1.48, \mathrm{p}=0.02)$ were associated with a similarly increased incidence of malaria compared to non-stunted children. Being mildly underweight (IRR $=1.09$, $95 \% \mathrm{Cl} 0.95-1.25, \mathrm{p}=0.24)$ and moderate-severe underweight (IRR $=1.12,95 \% \mathrm{Cl} 0.86-1.46, \mathrm{p}=0.39$ ) were not associated with a significant difference in the incidence of malaria compared to children who were not underweight. There were no significant interactions between HIV-infected, HIV-exposed children taking TS and the associations between malnutrition and the incidence of malaria.

Conclusions: Stunting, indicative of chronic malnutrition, was associated with an increased incidence of malaria among a cohort of HIV-infected and -uninfected young children living in an area of high malaria transmission intensity. However, caution should be made when making causal inferences given the observational study design and inability to disentangle the temporal relationship between malnutrition and the incidence of malaria.

Trial Registration: ClinicalTrials.gov: NCT00527800.

\footnotetext{
* Correspondence: emmy3md@yahoo.com

'Makerere University-University of California, San Francisco Research

Collaboration, Mulago Hospital Complex, PO Box 7475, Kampala, Uganda

Full list of author information is available at the end of the article
} 


\section{Background}

Malaria is a leading cause of childhood morbidity and mortality in sub-Saharan Africa [1]. According to the World Health Organization, in 2008 there were an estimated 243 million cases of malaria and 863,000 deaths of which $89 \%$ occurred in Africa and $88 \%$ of African deaths occurred in children under five years of age [2]. Malnutrition is also a major public health problem in African children, with estimated 38\% stunted, 28\% underweight, and 9\% wasted [3]. Malnutrition is responsible for an increased risk of a number of common childhood infectious diseases including diarrhoea and upper respiratory tract infections $[4,5]$, as well as increased case fatality $[4,6]$.

Findings from studies evaluating associations between various measures of malnutrition and malaria have been contradictory. Some studies suggest a protective effect against malaria for wasted children [7] and children with stunting $[8,9]$. In contrast, other studies have found either no association between various definitions of malnutrition and the risk of malaria [10-12], or an increased risk of malaria among stunted [13] and underweight children [14]. Comparisons across these studies are complicated by differences in study design, definitions of malnutrition and malaria morbidity, patient populations, and local malaria epidemiology.

The role that HIV exposure and infection may have on potential associations between malnutrition and malaria in children has not yet been reported. This is a critical gap because HIV infection has been associated with an increased risk of both malnutrition $[15,16]$ and malaria [17-20]. Further, because chemoprophylaxis with trimethoprim-sulphamethoxazole (TS) has become the standard of care for breastfeeding HIV-exposed infants (HIV-negative children born to HIV-infected mothers) and all HIV-infected children, and chemoprophylaxis with TS is protective against malaria $[21,22]$. Such an intervention may further modulate any potential interaction between HIV and associations between malnutrition and malaria.

To assess associations between paediatric malnutrition and the incidence of malaria by HIV status, prospective data were collected from a cohort of HIV-unexposed, HIV-exposed and HIV-infected children living in Tororo, a rural area in Uganda with high malaria transmission intensity [23].

\section{Methods}

\section{Study site and participants}

This study was conducted in the district of Tororo, a rural area in south-eastern Uganda. Malaria transmission in Tororo is holo-endemic, occurring perennially with an entomologic inoculation rate estimated to be 562 infective bites per person-year [23]. Data were collected from a cohort of HIV-unexposed, HIVexposed, and HIV-infected children as has been described elsewhere [24]. Briefly, convenience sampling was used to enrol children referred to a dedicated study clinic from an adjacent post-natal clinic at Tororo District Hospital. Eligibility criteria included: 1) age six weeks to 12 months; 2) documented HIV status of mother and child by DNA polymerase chain reaction (PCR); 3) agreement to come to the study clinic for any febrile episode or other illness; 4) residence within a 30 $\mathrm{km}$ radius of the study clinic; 5) absence of active medical problem requiring in-patient evaluation at the time of screening; 6) provision of informed consent; and 7) currently breastfeeding if HIV-exposed. At enrolment, all study participants received long-lasting insecticidetreated bed nets (ITN) and all HIV-exposed and HIVinfected children were given daily TS prophylaxis as per Uganda's Ministry of Health (MOH) guidelines. HIVunexposed children were not prescribed TS prophylaxis. The study protocol was approved by the Uganda National Council of Science and Technology and the institutional review boards of the University of California, San Francisco, Makerere University, the University of Washington, and the US Centers for Disease Control and Prevention.

\section{Follow-up of study participants}

Subjects were followed for all medical problems at a dedicated study clinic open seven days a week from 8 am to $5 \mathrm{pm}$. Parents (or guardians) were encouraged to bring their children to the study clinic whenever they were ill. After-hours care was available through the Tororo District Hospital. Children who presented with new medical problems underwent a standardized medical evaluation using algorithms to guide therapy for common illnesses that were developed locally by the study investigators. Medications with anti-malarial activity were avoided for the treatment of non-malarial illnesses. Monthly routine assessments were performed in the study clinic to ensure adherence with the study protocol. HIV-exposed children were re-tested for HIV by DNA PCR six to eight weeks after breastfeeding cessation. Those children who remained HIV-uninfected after breastfeeding cessation were then randomized to continue or discontinue TS prophylaxis as has been described elsewhere [25]. HIV-infected children, including those who seroconverted during breastfeeding, were continued on TS prophylaxis for the duration of the study. All HIV-infected children were anti-retroviral therapy (ART) naïve at enrolment and ART (nevirapine + lamivudine + zidovudine or stavudine) was initiated for those participants who met standardized WHO criteria. Study participants' compliance to TS, ART and ITN was assessed through questionnaires administered 
to their mothers at monthly visits. Study participants were not provided supplemental feeding. Study participants were withdrawn from the study for: 1) movement out of the study area; 2) inability to be located for $>60$ consecutive days; 3) withdrawal of informed consent; 4) inability to adhere to the study schedule and procedures; or 5) inability to tolerate the drugs used for malaria treatment.

\section{Anthropometric measurements}

Weight and length/height measurements were taken at every visit to the study clinic at least once a month by trained study nurses in accordance with internationally recommended procedures [26]. Participants were weighed using a designated baby scale (Seca- 310, Hamburg, Germany) precise to the nearest $0.1 \mathrm{~kg}$. Recumbent length measurements precise to the nearest centimetre were taken for children $<1$ year of age using a stadiometer board and standing height was measured for older children precise to the nearest centimetre using TALC height measuring chart (Teaching Aids at Low Cost, St Albans, UK).

\section{Malaria diagnosis and management}

Subjects who presented to the study clinic with a documented fever (tympanic temperature $\geq 38.0^{\circ} \mathrm{C}$ ) or history of fever in the previous 24 hours, had blood obtained by finger prick for a thick smear. If the thick smear was positive, the patient was diagnosed with malaria regardless of parasite density. Study participants $\geq 4$ months and $\geq 5 \mathrm{~kg}$ were randomly assigned to receive either open label artemether-lumefantrine (AL) or dihydroartemisinin-piperaquine (DP) at the time their first episode of uncomplicated malaria was diagnosed. Study participants received the same treatment regimen for all subsequent episodes of uncomplicated malaria. Episodes of uncomplicated malaria in children $<4$ months of age or weighing $<5 \mathrm{~kg}$, as well as episodes of complicated malaria and treatment failures occurring within 14 days of initiating treatment were treated with quinine.

\section{Laboratory methods}

Thick and thin blood smears were stained with $2 \%$ Giemsa for 30 minutes. A thick smear was considered negative if no parasites were seen after review of 100 high-powered fields. Speciation of parasites was based on readings of thin smears by a trained microscopist. A final diagnosis of malaria used in the analysis was based on a rigorous quality-control system that included rereading of all blood smears by a second microscopist and resolution of any discrepancies between the first and second readings by a third microscopist within 24 hours of the initial smear reading.
Assessment of infants HIV status prior to enrolment and at six to eight weeks after cessation of breastfeeding involved collecting a heel, toe or finger-prick blood sample on filter paper which was sent to a reference laboratory for HIV DNA PCR using Roche Amplicor HIV-1 DNA PCR test v1.5 kits (Roche Diagnostic Systems, Inc., Branchburg, New Jersey, USA).

\section{Statistical analysis}

Data were double entered in Access (Microsoft Corporation, Redmond, Washington, USA), and statistical analysis was performed using STATA, version 10 (Stata Corporation, College Station, Texas, USA). The observation period for each study participant began the day after enrolment and ended when a study participant was either prematurely withdrawn from the study or reached 2.5 years of age.

Nutritional status was assessed using the following standardized anthropometric z-scores as per WHO guidelines [27,28]: height-for-age $\mathrm{z}$ - score (HAZ) for stunting and weight-for-age $\mathrm{z}$-score (WAZ) for underweight. Measures of malnutrition were categorized as mild ( $z$-score between -1 and -2 ) and moderate-severe $(\mathrm{z}$-score $<-2)$. Wasting (based on weight-for-height $\mathrm{z}$ scores) was not included as a measure of nutritional status because values $<-1$ were relatively uncommon and therefore lacked statistical power for analyses. Repeated cross-sectional associations between variables of interest (breastfeeding, HIV-status, use of TS chemoprophylaxis and location of residence) and measures of malnutrition (dichotomized using $\mathrm{z}$-scores $<-1$ as a cut-off and expressed) were estimated as a relative risk using generalized estimating equations with exchangeable correlation and robust standard error, binomial distribution, and adjustment for repeated measures within individuals.

Malaria incidence was defined as the number of new episodes of malaria per time at risk. Episodes of malaria occurring within 14 days of a previous episode were not considered incident events. Longitudinal associations between variables of interest and malaria incidence were estimated as an incidence rate ratio using generalized estimating equations with exchangeable correlation, negative binomial distribution, and adjusted for repeated measures. For longitudinal associations, measures of malnutrition were categorized into none, mild, and moderate-severe based on the cut-offs above for mean z-scores during observation periods of interest. Interaction among potential confounders was also examined by including interaction terms into the multivariate models. A p-value $<0.05$ was considered statistically significant for all analyses.

\section{Results}

Study profile and characteristics of study participants

A total of 100 HIV-unexposed children, 203 HIVexposed children, and 48 HIV-infected children were 


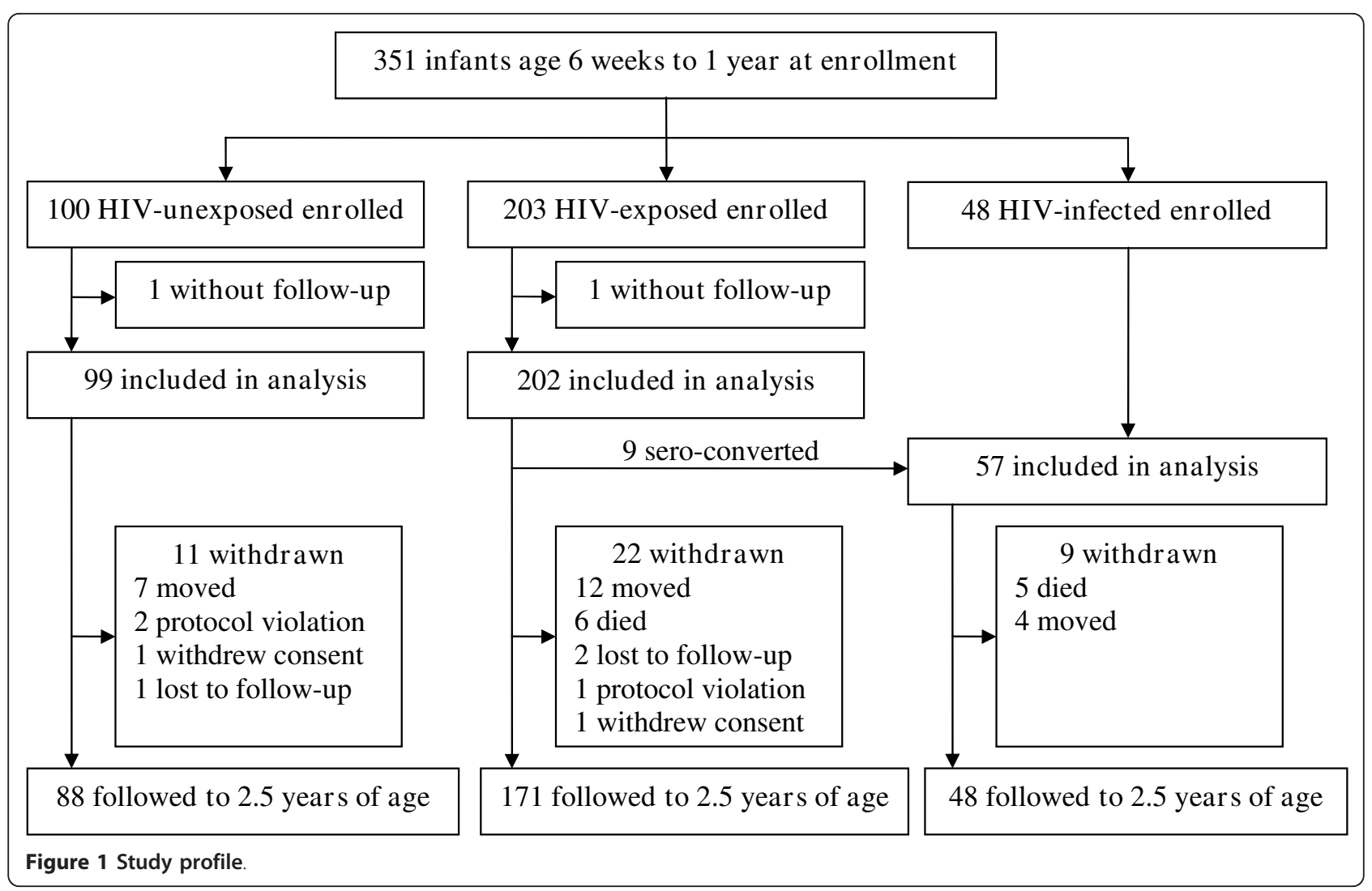

enrolled between August 2007 and April 2008 (Figure 1). Two participants with no follow-up information were not included in this analysis. During follow-up, nine HIV-exposed children seroconverted during breastfeeding resulting in a total of $57 \mathrm{HIV}$-infected children. Of the 349 study participants included in the study, a total of 307 (88\%) reached 2.5 years of age with the remaining 42 prematurely withdrawn.

Study participants were followed for mean of 16 months, resulting in a total observation period of 662 person-years. HIV-exposed children were prescribed TS for a total of $73 \%$ of the observation period (Table 1 ). Mothers of the HIV-infected and HIV-exposed children prescribed TS reported that their children took > 99\% of their doses. HIV-unexposed children breastfed until a median age of 20 months compared to 17 months among the HIV-infected children and seven months among the HIV-exposed children, whose mothers were counseled to rapidly wean after six months of age (per national recommendations at the time). During routine follow-up visits, mothers reported that over $98 \%$ of children slept under an ITN the previous night. All 57 HIV-infected children were ART naïve at enrolment. Fifty-two (91\%) were initiated on ART, two (3.5\%) were eligible but withdrew from the study before ART could be initiated, and three (5.2\%) never met eligibility criteria for ART initiation.

\section{Variables associated with measures of malnutrition}

Overall, $36 \%$ of study participants were mildly stunted and $34 \%$ were moderate-severely stunted. In contrast, $25 \%$ of study participants were mildly underweight and $13 \%$ were moderate-severely underweight. Mean HAZ scores decreased from six to 18 months of age (the age at which most children were stopping breastfeeding) and then rose slightly to 30 months of age (Figure 2). HAZ scores were consistently lower for HIV-infected children compared to HIV-uninfected children at all ages (Figure 2). Mean WAZ scores were fairly constant from six to 30 months of age and lower for HIVinfected children, particularly in younger children.

In multi-variate analysis, both breastfeeding ( $R R=$ $0.75,95 \%$ CI $0.69-0.82, \mathrm{p}<0.001)$ and being prescribed TS chemoprophylaxis $(\mathrm{RR}=0.82,95 \%$ CI $0.74-0.90, \mathrm{p}<$ 0.001 ) were associated with a lower risk of stunting of any severity (Table 2). HIV-infection was associated with a higher risk of stunting $(\mathrm{RR}=1.38,95 \% \mathrm{CI} 1.18$ $1.62, \mathrm{p}<0.001)$. The only significant risk factor for being underweight of any severity was HIV-infection $(\mathrm{RR}=1.41,95 \%$ CI 1.02-1.96, $\mathrm{p}<0.001)$. 
Table 1 Characteristics of study participants

\begin{tabular}{|c|c|c|c|}
\hline \multirow[t]{2}{*}{ Characteristic } & \multicolumn{3}{|c|}{ Risk group } \\
\hline & HIV-unexposed $(n=99)$ & HIV-exposed $(n=202)$ & HIV-infected $(n=57)$ \\
\hline Female gender, n (\%) & $40(40 \%)$ & $101(50 \%)$ & $28(49 \%)$ \\
\hline Residence in urban area, $\mathrm{n}(\%)$ & $23(23 \%)$ & $39(19 \%)$ & $18(32 \%)$ \\
\hline Median duration of follow-up, months (IQR) & $15.2(12.1-17.5)$ & $16.7(13.4-19.5)$ & $14.3(11.8-18.4)$ \\
\hline Mean age during follow-up, months (SD) & $14.3(2.0)$ & $13.2(2.8)$ & $14.3(3.1)$ \\
\hline Proportion of follow-up time prescribed TS & 0 & $73 \%$ & $100 \%$ \\
\hline Proportion of follow-up time breastfeeding & $81 \%$ & $24 \%$ & $64 \%$ \\
\hline \multicolumn{4}{|l|}{ Mean HAZ scores } \\
\hline Mild stunting, n (\%) & $38(38 \%)$ & $71(35 \%)$ & $17(30 \%)$ \\
\hline Moderate-severe stunting, n (\%) & $28(28 \%)$ & $69(34 \%)$ & $29(51 \%)$ \\
\hline \multicolumn{4}{|l|}{ Mean WAZ scores } \\
\hline Mild underweight & $23(23 \%)$ & $52(26 \%)$ & $13(23 \%)$ \\
\hline Moderate-severe underweight & $7(7.1 \%)$ & $21(10 \%)$ & $17(30 \%)$ \\
\hline
\end{tabular}

\section{Variables associated with the incidence of malaria}

By the end of the observation period, 288 (83\%) of the study participants had experienced at least one episode of malaria. A total of 2,414 new episodes of malaria were diagnosed, resulting in an overall incidence of 3.64 episodes per person year. In multi-variate analysis, children with mild stunting $(\mathrm{IRR}=1.24,95 \%$ CI 1.06-1.46, $\mathrm{p}=0.008)$ and moderate-severe stunting (IRR $=1.24$, $95 \%$ CI 1.03-1.48, $\mathrm{p}=0.02$ ) had a similarly higher incidence of malaria compared to children without stunting (Table 3 ). The association between stunting and an increased incidence of malaria was similar for HIVinfected and uninfected children ( $\mathrm{p}=0.52$ for interaction term in multi-variate model). As expected, being prescribed TS prophylaxis and living in an urban residence were associated with a significantly lower incidence of malaria. Notably, breastfeeding was associated with a lower incidence of malaria in the uni-variate analysis, but not in the multi-variate analysis. Increasing age was associated with a significantly higher incidence of malaria. On the other hand, HIV infection was not associated with the incidence of malaria in the multivariate analysis (Table 3 ). In contrast, being mildly underweight $(\mathrm{IRR}=1.09,95 \%$ CI $0.95-1.25, \mathrm{p}=0.24)$ and moderate-severe underweight $(\mathrm{IRR}=1.12,95 \% \mathrm{CI}$ $0.86-1.46, \mathrm{p}=0.39$ ) were not associated with a significant difference in the incidence of malaria compared to children who were not underweight.

\section{Discussion}

This study was conducted in a cohort of young children living in an area of high malaria transmission intensity, where the incidence of malaria was over 3.6 episodes per person-year despite the use of long-lasting ITNs by all study participants and TS prophylaxis in majority of the study participants. Malnutrition was also very common,

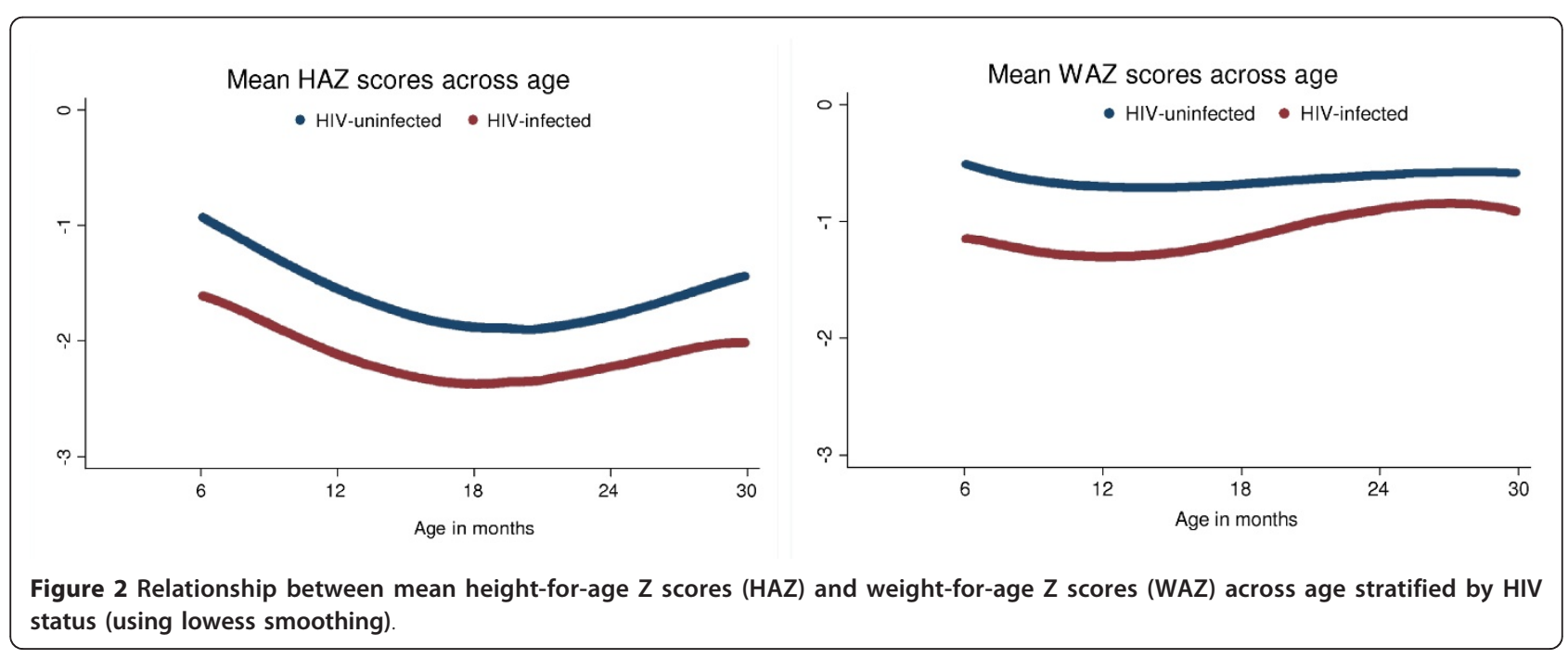


Table 2 Variables associated with measures of malnutrition

\begin{tabular}{|c|c|c|c|c|c|c|}
\hline \multirow[t]{2}{*}{ Variable } & \multicolumn{2}{|c|}{ Proportion with z-score $<-1$} & \multirow[t]{2}{*}{ Unadjusted $\mathrm{RR}^{*}(95 \% \mathrm{Cl})$} & \multirow[t]{2}{*}{ p-value } & \multirow[t]{2}{*}{ Adjusted $\mathrm{RR}^{* *}(95 \% \mathrm{Cl})$} & \multirow[t]{2}{*}{ p-value } \\
\hline & Exposed group $^{+}$ & Unexposed group & & & & \\
\hline \multicolumn{7}{|c|}{ Stunting (HAZ-score) } \\
\hline Breastfeeding & $52 \%$ & $73 \%$ & $0.70(0.64-0.76)$ & $<0.001$ & $0.75(0.69-0.82)$ & $<0.001$ \\
\hline HIV-infected & $79 \%$ & $62 \%$ & $1.34(1.16-1.54)$ & $<0.001$ & $1.38(1.18-1.62)$ & $<0.001$ \\
\hline TS chemoprophylaxis & $59 \%$ & $71 \%$ & $0.78(0.71-0.85)$ & $<0.001$ & $0.82(0.74-0.90)$ & $<0.001$ \\
\hline Urban vs. rural residence & $57 \%$ & $65 \%$ & $0.87(0.73-1.04)$ & 0.12 & $0.87(0.74-1.03)$ & 0.10 \\
\hline \multicolumn{7}{|c|}{ Being underweight (WAZ-score) } \\
\hline Breastfeeding & $34 \%$ & $37 \%$ & $0.89(0.78-1.01)$ & 0.08 & $0.89(0.78-1.02)$ & 0.09 \\
\hline HIV infected & $49 \%$ & $34 \%$ & $1.48(1.09-2.01)$ & 0.01 & $1.41(1.02-1.96)$ & 0.04 \\
\hline TS chemoprophylaxis & $37 \%$ & $34 \%$ & $1.06(0.90-1.25)$ & 0.49 & $1.06(0.89-1.27)$ & 0.52 \\
\hline Urban vs. rural residence & $33 \%$ & $36 \%$ & $0.91(0.66-1.26)$ & 0.57 & $0.88(0.65-1.21)$ & 0.45 \\
\hline
\end{tabular}

with $70 \%$ of study participants meeting criteria for mild stunting, and $38 \%$ of study participants being mildly underweight. HIV infection was associated with an increased risk of both stunting and being underweight, while breastfeeding and the use of TS prophylaxis were protective against stunting, but not being underweight.

The main objective of the study was to evaluate associations between paediatric malnutrition and the incidence of malaria by HIV status. Stunting was associated with a modest $24 \%$ increase in the incidence of malaria after controlling for potential confounding factors, but the degree of stunting did not modulate this association. In addition, the association between stunting and an increased incidence of malaria was consistent between HIV-infected and uninfected children. In contrast, there was no statistically significant association between being underweight and the incidence of malaria.

Interactions between malnutrition and HIV infection are complex and have been suggested to have negative feedback loops. Malnutrition is a significant risk factor for AIDS-related mortality and HIV-associated wasting often persists even with use of ART [29,30]. The direct effect of HIV on impaired metabolic function in absorption, storage and utilization of nutrients can translate into compromised immunity, nutrient deficiencies and increased vulnerability to infectious diseases [5]. In this study, HIV-infected children were more likely to be malnourished as compared to the HIV-uninfected children, which is in accord with the existing literature. The finding from this study that breastfeeding is protective against malnutrition is also consistent with the existing literature [31,32] and highlights the importance of breastfeeding promotion as one of the most cost-effective and feasible child survival interventions [33].

Another novel finding of this study was that children taking TS prophylaxis had a significantly lower risk of stunting. A similar finding was reported in a randomized control trial of TS prophylaxis among HIV-

Table 3 Variables associated with the incidence of malaria

\begin{tabular}{|c|c|c|c|c|c|c|}
\hline \multirow[t]{2}{*}{ Variable } & \multicolumn{2}{|c|}{ Incidence of malaria } & \multirow[t]{2}{*}{ Unadjusted IRR* $(95 \% \mathrm{Cl})$} & \multirow[t]{2}{*}{$p$-value } & \multirow[t]{2}{*}{ Adjusted IRR** $(95 \% \mathrm{Cl})$} & \multirow[t]{2}{*}{ p-value } \\
\hline & Exposed $^{\dagger}$ & Unexposed & & & & \\
\hline \multicolumn{7}{|l|}{ Measures of stunting } \\
\hline none & - & 2.87 & reference & - & reference & - \\
\hline mild & 4.02 & - & $1.55(1.31-1.83)$ & $<0.001$ & $1.24(1.06-1.46)$ & -0.008 \\
\hline moderate-severe & 3.89 & - & $1.64(1.37-1.98)$ & $<0.001$ & $1.24(1.03-1.48)$ & 0.02 \\
\hline Breastfeeding & 2.93 & 4.02 & $0.56(0.50-0.63)$ & $<0.001$ & $0.85(0.68-1.07)$ & 0.17 \\
\hline HIV infected & 2.06 & 3.91 & $0.56(0.43-0.75)$ & $<0.001$ & $0.93(0.67-1.30)$ & 0.68 \\
\hline TS chemoprophylaxis & 2.18 & 5.05 & $0.38(0.33-0.43)$ & $<0.001$ & $0.50(0.41-0.61)$ & $<0.001$ \\
\hline Urban vs. rural residence & 1.65 & 4.19 & $0.41(0.31-0.52)$ & $<0.001$ & $0.44(0.34-0.56)$ & $<0.001$ \\
\hline Age per 1 year increase & \multicolumn{2}{|c|}{ N/A } & $2.22(2.01-2.44)$ & $<0.001$ & $1.49(1.20-1.84)$ & $<0.001$ \\
\hline
\end{tabular}

${ }^{\dagger}$ Exposed groups include stunted, breastfeeding, HIV infected participants, TS chemoprophylaxis, and urban residence

* IRR incidence rate ratio using univariate analysis including only the variable of interest

**IRR incidence rate ratio using multivariate analysis including all variables of interest listed in the table 
infected adults in Côte d'Ivoire, suggesting that TS prophylaxis may improve nutritional status by the prevention of morbidity-related malnutrition or by other indirect mechanisms [34].

Associations between measures of malnutrition and the risk of malaria appear to be complex as previous studies have reported a wide range of findings. One source of this complexity is due to differences in the indicators commonly used for malnutrition. Stunting is generally considered an indicator of chronic malnutrition, wasting generally reflects a recent and severe process, and underweight a reflection of a combination of factors. Earlier studies provide some evidence of a protective effect of stunting against malaria $[8,35]$ and the presence of malaria in famine victims within few days of re-feeding suggested that feeding provided essential nutrients for sequestered parasites leading to recrudescent infection $[9,36]$. More recently in a cohort of preschool children from Senegal, those who were wasted had $67 \%$ lower odds of having at least one subsequent clinical malaria attack, whereas stunting and being underweight were not associated with clinical malaria [7]. In contrast, several studies have reported an increased risk of malaria among stunted children. In a large cross-sectional study from Ghana, children who were underweight had $67 \%$ increased odds of clinical malaria [14]. In a prospective cohort study of Gambian children, those with baseline stunting had a 35\% higher risk of experiencing at least one episode of malaria during rainy season [13], yet neither wasting nor being underweight influenced susceptibility to malaria. The results presented in this report support the finding that stunting, a measure of chronic malnutrition is associated with an increased risk of malaria.

There are several hypotheses as to why chronic malnutrition may increase the risk of malaria. It is now widely known that nutrition plays an important role in modulation of anti-pathogen immunity and most studies have demonstrated a deleterious effect of malnutrition on the appropriate immune response to infection $[37,38]$. In addition, given that chronic malnutrition is frequently accompanied by nutritional deficiencies, it is conceivable that multiple specific nutrients may influence malaria infection and pathology $[39,40]$.

Strengths of this study include the prospective, longitudinal study design and the assessment of HIV status. The mean of anthropometric measures of malnutrition taken over an extended period of follow-up were obtained and therefore better reflect the long-term nutritional status of a child compared to studies which rely on single measurements. In addition, malaria morbidity was analysed using an incidence measure of clinical disease, which best reflects the repeated nature of malaria in children residing in high transmission areas.
Another strength is the inclusion of HIV-exposed and infected children, which has not been previously reported in studies evaluating associations between malnutrition and malaria. Limitations of this study include the lack of assessment of the temporal relationship between malnutrition and malaria. A number of studies have indicated a deleterious effect of malaria on nutritional status $[41,42]$. Therefore, in this study it is possible that malaria itself could have been in the causal pathway of the observed association between stunting and an increased incidence of malaria. The relatively low number of children with wasting in this study was also a limitation and precluded the ability to evaluate this measure of malnutrition as a risk factor for malaria. Finally, the presence of unmeasured confounders that could explain the observed association between stunting and an increased incidence of malaria cannot be ruled out.

\section{Conclusion}

In this study of young children living in a high transmission area, stunting was associated with an increased risk of malaria regardless of HIV status. Of note, the incidence of malaria was high in this cohort despite the use of ITNs and TS prophylaxis in a substantial number of children. Intervention studies aimed at improving nutritional status as a means of reducing the incidence of malaria in similar epidemiological settings deserve exploration.

\section{Acknowledgements}

The authors gratefully appreciate the children who participated in this study as well as their parents and guardians. Subjects in this study were enrolled in programmes supported by the US President's Emergency Plan for AIDS Relief Cooperative Agreement Number U62P024421 from the Department of Health and Human Services/Centers for Disease Control and Prevention (CDC), National Center for HIV, Viral Hepatitis, STD, and TB Prevention (NCHHSTP), Global AIDS Program (GAP). Funding was also provided by the Doris Duke Charitable Foundation (GD is a recipient of the Clinical Scientist Development Award) and training support from the Fogarty AIDS International Research and Training grant (1 D43 TW00003). This project was partially supported by the Uganda Malaria Clinical Operational and Health Services (COHRE) Training Program at Makerere University, Grant \#D43TW00807701A1, funded by the Fogarty International Center of the National Institutes of Health.

The findings and conclusions in this paper are those of the authors and do not necessarily represent the views of the Centers for Disease Control and Prevention.

\section{Author details}

${ }^{1}$ Makerere University-University of California, San Francisco Research Collaboration, Mulago Hospital Complex, PO Box 7475, Kampala, Uganda. ${ }^{2}$ Department of Epidemiology, University of California, Berkeley, CA, USA. ${ }^{3}$ Global Health Sciences, University of California, San Francisco, CA, USA. ${ }^{4}$ Department of Medicine, University of Washington, Seattle, WA, USA ${ }^{5}$ Division of Nutritional Sciences, Cornell University, Ithaca, NY, USA. ${ }^{6}$ Center for Global Health, Centers for Disease Control and Prevention, Atlanta, GA, USA. ${ }^{7}$ Department of Medicine, Makerere University College of Health Sciences, Kampala, Uganda. ${ }^{8}$ Department of Medicine, University of California, San Francisco, CA, USA. 


\section{Authors' contributions}

EA, TGS, JH, HW, AK, JT, MRK and GD contributed to the study design and oversight. EA, AG, WV, SY and GD contributed to the methodology, data analysis, interpretation of the results and drafting of the manuscript. All authors read and reviewed the final manuscript. All authors read and approved the final manuscript.

\section{Competing interests}

The authors declare that they have no competing interests.

Received: 7 January 2012 Accepted: 27 March 2012

Published: 27 March 2012

\section{References}

1. Rosen JB, Breman JG: Malaria intermittent preventive treatment in infants, chemoprophylaxis, and childhood vaccinations. Lancet 2004, 363:1386-1388

2. World Health Organization (WHO): World Malaria Report 2009 Geneva, Switzerland: World Health Organization; 2009.

3. Progress for Children: A World Fit for Children: Statistical Review New York: United Nations; 2007, 19-24.

4. Caulfield LE, Richard SA, Black RE: Undernutrition as an underlying cause of malaria morbidity and mortality in children less than five years old. Am J Trop Med Hyg 2004, 71:55-63.

5. Katona $\mathrm{P}$, Katona-Apte J: The interaction between nutrition and infection. Clin Infect Dis 2008, 46:1582-1588.

6. Pelletier DL, Frongillo EA, Schroeder DG, Habicht JP: The effects of malnutrition on child mortality in developing countries. Bull World Health Organ 1995, 73:443-448.

7. Fillol F, Cournil A, Boulanger D, Cisse B, Sokhna C, Targett G, Trape JF, Simondon F, Greenwood B, Simondon KB: Influence of wasting and stunting at the onset of the rainy season on subsequent malaria morbidity among rural preschool children in Senegal. Am J Trop Med Hyg 2009, 80:202-208.

8. Genton B, Al-Yaman F, Ginny M, Taraika J, Alpers MP: Relation of anthropometry to malaria morbidity and immunity in Papua New Guinean children. Am J Clin Nutr 1998, 68:734-741.

9. Murray MJ, Murray AB, Murray MB, Murray CJ: Somali food shelters in the Ogaden famine and their impact on health. Lancet 1976, 1:1283-1285.

10. Carswell F, Hughes AO, Palmer RI, Higginson J, Harland PS, Meakins RH: Nutritional status, globulin titers, and parasitic infections of two populations of Tanzanian school children. Am J Clin Nutr 1981, 34:1292-1299.

11. Snow RW, Byass P, Shenton FC, Greenwood BM: The relationship between anthropometric measurements and measurements of iron status and susceptibility to malaria in Gambian children. Trans $R$ Soc Trop Med Hyg 1991, 85:584-589.

12. Deribew A, Alemseged F, Tessema F, Sena L, Birhanu Z, Zeynudin A, Sudhakar M, Abdo N, Deribe K, Biadgilign S: Malaria and under-nutrition: a community based study among under-five children at risk of malaria, south-west Ethiopia. PLoS One 2010, 5:e10775

13. Deen $J$, Walraven $G E$, von Seidlein $L$ : Increased risk for malaria in chronically malnourished children under 5 years of age in rural Gambia. J Trop Pediatr 2002, 48:78-83.

14. Ehrhardt S, Burchard GD, Mantel C, Cramer JP, Kaiser S, Kubo M, Otchwemah RN, Bienzle U, Mockenhaupt FP: Malaria, anemia, and malnutrition in african children-defining intervention priorities. J Infect Dis 2006, 194:108-114.

15. Suttmann U, Ockenga J, Selberg O, Hoogestraat L, Deicher H, Muller MJ: Incidence and prognostic value of malnutrition and wasting in human immunodeficiency virus-infected outpatients. J Acquir Immune Defic Syndr Hum Retrovirol 1995, 8:239-246.

16. Padmapriyadarsini C, Pooranagangadevi N, Chandrasekaran $\mathrm{K}$, Subramanyan S, Thiruvalluvan C, Bhavani PK, Swaminathan S: Prevalence of underweight, stunting, and wasting among children infected with human immunodeficiency virus in South India. Int J Pediatr 2009, 200(9):837627.

17. Whitworth J, Morgan D, Quigley M, Smith A, Mayanja B, Eotu H, Omoding N, Okongo M, Malamba S, Ojwiya A: Effect of HIV-1 and increasing immunosuppression on malaria parasitaemia and clinical episodes in adults in rural Uganda: a cohort study. Lancet 2000, 356:1051-1056.

18. French N, Nakiyingi J, Lugada E, Watera C, Whitworth JA, Gilks CF: Increasing rates of malarial fever with deteriorating immune status in HIV-1-infected Ugandan adults. AIDS 2001, 15:899-906.

19. Kamya MR, Gasasira AF, Yeka A, Bakyaita N, Nsobya SL, Francis D, Rosenthal PJ, Dorsey G, Havlir D: Effect of HIV-1 infection on antimalarial treatment outcomes in Uganda: a population-based study. I Infect Dis 2006, 193:9-15.

20. Patnaik P, Jere CS, Miller WC, Hoffman IF, Wirima J, Pendame R, Meshnick SR, Taylor TE, Molyneux ME, Kublin JG: Effects of HIV-1 serostatus, HIV-1 RNA concentration, and CD4 cell count on the incidence of malaria infection in a cohort of adults in rural Malawi. $J$ Infect Dis 2005, 192:984-991.

21. Mermin J, Ekwaru JP, Liechty CA, Were W, Downing R, Ransom R, Weidle P, Lule J, Coutinho A, Solberg P: Effect of co-trimoxazole prophylaxis, antiretroviral therapy, and insecticide-treated bednets on the frequency of malaria in HIV-1-infected adults in Uganda: a prospective cohort study. Lancet 2006, 367:1256-1261.

22. Kamya MR, Gasasira AF, Achan J, Mebrahtu T, Ruel T, Kekitiinwa A, Charlebois ED, Rosenthal PJ, Havlir D, Dorsey G: Effects of trimethoprimsulfamethoxazole and insecticide-treated bednets on malaria among HIV-infected Ugandan children. AIDS 2007, 21:2059-2066.

23. Okello PE, Van Bortel W, Byaruhanga AM, Correwyn A, Roelants $P$, Talisuna A, D'Alessandro U, Coosemans M: Variation in malaria transmission intensity in seven sites throughout Uganda. Am J Trop Med Hyg 2006, 75:219-225.

24. Arinaitwe E, Sandison TG, Wanzira H, Kakuru A, Homsy J, Kalamya J, Kamya MR, Vora N, Greenhouse B, Rosenthal PJ, Tappero J, Dorsey G: Artemether-lumefantrine versus dihydroartemisinin-piperaquine for falciparum malaria: a longitudinal, randomized trial in young Ugandan children. Clin Infect Dis 2009, 49:1629-1637.

25. Sandison TG, Homsy J, Arinaitwe E, Wanzira H, Kakuru A, Bigira V, Kalamya J, Vora N, Kublin J, Kamya MR, Dorsey G, Tappero JW: Protective efficacy of co-trimoxazole prophylaxis against malaria in HIV exposed children in rural Uganda: a randomised clinical trial. BMJ 2011, 342:d1617.

26. Bates B: Bate's Guide to Physical Examination and History Taking. 7 edition. Philadelphia: Lippincott; 1999

27. Cogill B: Anthropometric Indicators Measurement Guide. Food and Nutrition Technical Assistant 2003.

28. World Health Organization (WHO) Multicentre Growth Reference Study Group: WHO Child Growth Standards: Methods and Development. Geneva, Switzerland: World Health Organization; 2006.

29. Mangili A, Murman DH, Zampini AM, Wanke CA: Nutrition and HIV infection: review of weight loss and wasting in the era of highly active antiretroviral therapy from the nutrition for healthy living cohort. Clin Infect Dis 2006, 42:836-842.

30. Tang AM, Forrester J, Spiegelman D, Knox TA, Tchetgen E, Gorbach SL: Weight loss and survival in HIV-positive patients in the era of highly active antiretroviral therapy. J Acquir Immune Defic Syndr 2002, 31:230-236.

31. Arpadi S, Fawzy A, Aldrovandi GM, Kankasa C, Sinkala M, Mwiya M, Thea DM, Kuhn L: Growth faltering due to breastfeeding cessation in uninfected children born to HIV-infected mothers in Zambia. Am J Clin Nutr 2009, 90:344-353.

32. Mane NB, Simondon KB, Diallo A, Marra AM, Simondon F: Early breastfeeding cessation in rural Senegal: causes, modes, and consequences. Am J Public Health 2006, 96:139-144.

33. World Health Organization: Effect of breastfeeding on infant and child mortality due to infectious diseases in less developed countries: a pooled analysis. Lancet 2000, 355:451-455.

34. Castetbon $K$, Anglaret $X$, Toure $S$, Chene G, Ouassa T, Attia A, N'DriYoman T, Malvy D, Salamon R, Dabis F: Prognostic value of cross-sectional anthropometric indices on short-term risk of mortality in human immunodeficiency virus-infected adults in Abidjan, Cote d'Ivoire. Am J Epidemiol 2001, 154:75-84.

35. Hendrickse RG, Hasan AH, Olumide LO, Akinkunmi A: Malaria in early childhood. An investigation of five hundred seriously ill children in whom a "clinical" diagnosis of malaria was made on admission to the children's emergency room at University College Hospital, Ibadan. Ann Trop Med Parasitol 1971, 65:1-20. 
36. Murray MJ, Murray NJ, Murray AB, Murray MB: Refeeding-malaria and hyperferraemia. Lancet 1975, 1:653-654.

37. Cunningham-Rundles SD, McNeeley F, Moon A: Mechanisms of nutrient modulation of the immune response. J Allergy Clin Immunol 2005, 115:1119-1128.

38. Field CJ, Johnson IR, Schley PD: Nutrients and their role in host resistance to infection. J Leukoc Biol 2002, 71:16-32.

39. Semba RD: The role of vitamin $A$ and related retinoids in immune function. Nutr Rev 1998, 56:S38-S48.

40. Shankar AH, Prasad AS: Zinc and immune function: the biological basis of altered resistance to infection. Am J Clin Nutr 1998, 68:447S-463S.

41. Bradley-Moore AM, Greenwood BM, Bradley AK, Kirkwood BR, Gilles HM: Malaria chemoprophylaxis with chloroquine in young Nigerian children. III. Its effect on nutrition. Ann Trop Med Parasitol 1985, 79:575-584.

42. Snow RW, Molyneux CS, Njeru EK, Omumbo J, Nevill CG, Muniu E, Marsh K: The effects of malaria control on nutritional status in infancy. Acta Trop 1997, 65:1-10.

doi:10.1186/1475-2875-11-90

Cite this article as: Arinaitwe et al:: The association between malnutrition and the incidence of malaria among young HIV-infected and -uninfected Ugandan children: a prospective study. Malaria Journal 2012 11:90.

\section{Submit your next manuscript to BioMed Central and take full advantage of:}

- Convenient online submission

- Thorough peer review

- No space constraints or color figure charges

- Immediate publication on acceptance

- Inclusion in PubMed, CAS, Scopus and Google Scholar

- Research which is freely available for redistribution

Submit your manuscript at www.biomedcentral.com/submit 